\title{
Salvia hispanica Seed
}

National Cancer Institute

\section{Source}

National Cancer Institute. Salvia hispanica Seed. NCI Thesaurus. Code C124994.

The edible seed of the flowering plant Salvia hispanica (chia) used as a nutritional supplement, with potential immunomodulating activity. Upon ing estion, chia seed supplies essential fatty acids, including alpha linolenic acid (an omega-3) and linoleic acid (an omega-6), B vitamins, especially niacin (B3) and thiamine (B1), and several minerals, including calcium, zinc, mang anese, magnesium, phosphorus and iron; it also contains high levels of antioxidants and dietary soluble fiber. When used as a dietary supplement, this agent may improve a patient's nutrient intake and may balance their intestinal microbiome. 